\title{
The number of Dirac-weighted eigenvalues of Sturm-Liouville equations with integrable potentials and an application to inverse problems
}

\author{
Xiao Chen ${ }^{1}$ and Jiangang Qi ${ }^{1}$ \\ ${ }^{1}$ Shandong University at Weihai
}

January 10, 2021

\begin{abstract}
In this paper, we further Meirong Zhang, et al.'s work by computing the number of weighted eigenvalues for Sturm-Liouville equations, equipped with general integrable potentials and Dirac weights, under Dirichlet boundary condition. We show that, for a Sturm-Liouville equation with a general integrable potential, if its weight is a positive linear combination of $\$ \mathrm{n} \$ \mathrm{Dirac}$ Delta functions, then it has at most $\$ \mathrm{n} \$$ (may be less than $\$ \mathrm{n} \$$, or even be $\$ 0 \$$ ) distinct real Dirichlet eigenvalues, or every complex number is a Dirichlet eigenvalue; in particular, under some sharp condition, the number of Dirichlet eigenvalues is exactly $\$ \mathrm{n} \$$. Our main method is to introduce the concepts of characteristics matrix and characteristics polynomial for SturmLiouville problem with Dirac weights, and put forward a general and direct algorithm used for computing eigenvalues. As an application, a class of inverse Dirichelt problems for Sturm-Liouville equations involving single Dirac distribution weights is studied.
\end{abstract}

\section{Hosted file}

Chen_Qi_number_eigenvalues_SLeqn_inverse_problems.pdf available at https://authorea. com/users/388508/articles/503294-the-number-of-dirac-weighted-eigenvalues-of-sturmliouville-equations-with-integrable-potentials-and-an-application-to-inverse-problems 